\section{Histoire Épistémologie Langage}

42-1 | 2020

La grammaire arabe étendue

\title{
Les options de catégorisation du participe des temps composés dans les grammaires des langues romanes $\left(\mathrm{XV}^{\mathrm{e}}-\mathrm{XVIII}{ }^{\mathrm{e}}\right.$ siècles)
}

\section{Alejandro Díaz Villalba}

\section{Q OpenEdition}

\section{Journals}

Édition électronique

URL : https://journals.openedition.org/hel/594

DOI : $10.4000 /$ hel. 594

ISSN : 1638-1580

Éditeur

Société d'histoire et d'épistémologie des sciences du langage

Édition imprimée

Date de publication : 28 septembre 2020

Pagination : 135-154

ISSN : 0750-8069

\section{Référence électronique}

Alejandro Díaz Villalba, «Les options de catégorisation du participe des temps composés dans les

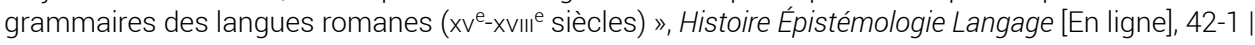
2020, mis en ligne le 28 octobre 2021, consulté le 29 octobre 2021. URL : http://

journals.openedition.org/hel/594; DOl : https://doi.org/10.4000/hel.594

Ce document a été généré automatiquement le 29 octobre 2021.

\section{(c) (i) $\odot$}

HEL is licensed under a Creative Commons Attribution-NonCommercial-NoDerivatives 4.0 International License 


\title{
Les options de catégorisation du participe des temps composés dans les grammaires des langues romanes $\left(\mathrm{XV}^{\mathrm{e}}-\mathrm{XVIII} \mathrm{e}^{\mathrm{e}}\right.$ siècles)
}

\author{
Alejandro Díaz Villalba
}

\section{Préambule}

\subsection{Les temps composés : le modèle latin face aux données des langues vernaculaires}

1 La série des tiroirs verbaux appelés communément «temps composés » (désormais TC) constitue un phénomène morphologique commun aux différentes langues romanes, $\mathrm{du}$ moins pour le type avoir + participe (j'ai chanté), car le type être + participe (je suis arrivé) ne subsiste aujourd'hui que dans une partie des langues, comme le français et l'italien. La coalescence de avoir+participe, pour exprimer des contenus temporels et aspectuels, fait figure d'innovation à l'égard du latin classique. Si une telle structure y est attestée en tant que périphrase verbale résultative, l'association de avoir au "participe des temps composés» (désormais PTC) a été vraiment exploitée dans les langues modernes, qui ont restructuré de fond en comble le système verbal.

Lorsque la grammatisation des langues romanes, s'appuyant sur les grammaires latines, émerge aux $\mathrm{xv}^{\mathrm{e}}$ et $\mathrm{xvI}^{\mathrm{e}}$ siècles, l'existence des formes verbales composées est une donnée déjà bien en place dans ces langues. Ainsi, l'examen du traitement des TC constitue un excellent terrain d'observation pour étudier les modalités du transfert du cadre conceptuel et explicatif du latin vers le traitement des vernaculaires. En d'autres termes, il s'agit d'observer la manière dont les grammairiens de la Renaissance s'emparent du modèle disponible, élaboré pour le latin, et initialement pour le grec, afin de traiter d'objets nouveaux. 


\subsection{Une histoire sérielle et comparée}

3 Le corpus est composé de 97 grammaires examinées dans le cadre d'une recherche approfondie sur l'histoire du participe (Díaz Villalba 2017). Il réunit des œuvres parues entre 1400 et 1800 qui décrivent l'espagnol, le français, l'italien et le portugais, ainsi que des grammaires générales rédigées dans ces langues. Cet ensemble de textes invite à la comparaison, dans la mesure où les langues entretiennent un double lien de parenté : un lien interne ou linguistique, puisque toutes dérivent du latin et partagent bon nombre de faits de langue; un lien externe ou épistémologique, parce que leur codification se réalise dans le même moule théorique et descriptif, la grammaire latine.

4 L'histoire comparée se fonde, dans cette étude, sur le croisement des traditions. C'est précisément le socle grammatical partagé par les traditions qui assure la possibilité de mettre les textes en parallèle.

Nous faisons le choix d'une histoire sérielle. Le concept de série textuelle recouvre souvent un regroupement cohérent : « un ensemble de textes imprimés ou manuscrits qui traitent le même sujet dans le même cadre épistémologique ou bien sans méthode déclarée, mais avec le même but et dans des conditions comparables. » (Haßler 2000, p. 97). Or, la mise en série est conçue, dans notre étude, de manière plus restreinte : elle concerne la réunion de textes qui abordent le même problème en proposant des solutions théoriques et des innovations terminologiques comparables, en dépit de leur appartenance à des traditions linguistiques et des projets scientifiques hétérogènes. Dans les deux démarches, il existe une construction historienne, la seconde impliquant un changement d'échelle historiographique et posant foncièrement le problème de la commensurabilité.

\section{La délimitation des problèmes et leur traitement dans les grammaires}

6 Il convient de délimiter les objets linguistiques que les grammairiens se proposent de réduire à un cadre intelligible; il importe également de présenter les procédés théoriques et explicatifs qu'ils mobilisent. Les phénomènes qui mettent à l'épreuve les ressources descriptives des premiers grammairiens ( $\mathrm{XV}^{\mathrm{e}}$-XVII ${ }^{\mathrm{e}}$ siècles) peuvent être résumés en trois questions :

\subsection{Le caractère analytique des formes verbales}

7 Un des aspects abordés par les premiers grammairiens est le caractère analytique d'une partie de la conjugaison verbale, plus particulièrement l'expression de la diathèse passive (je suis aimé) et celle de la diathèse active (j'ai aimé, je suis venu). Il importe de souligner deux faits remarquables dans ce que l'on pourrait appeler une approche périphrastique du verbe: d'une part, la prégnance de l'organisation binaire (temps simple vs temps double) de la morphologie vernaculaire, dont la manifestation la plus aboutie apparaît dans les chapitres consacrés aux formes périphrastiques chez Nebrija (1981 [1492] : 187-188), Barros (1540, f. 25r-26r), l'Anonyme de Louvain (1966 [1559] : 55) et chez Ramus (1562: 100-107); d'autre part, la variation terminologique pour exprimer le caractère composé. Les désignations les plus fréquentes sont en latin 
circumlocutio, circumloquor, en français circonlocution, périphrase (Fournier \& Raby 2014), en espagnol et en portugais circunloquio, rodeo (Gómez Asencio 2015), mais des verbes comme suppléer et ses cognats romans sont également courants. L'extension des objets désignés par ces termes n'est pas toujours homogène, bien que l'on trouve des récurrences (outre les formes verbales analytiques, on y place souvent les degrés de l'adjectif et le verbe impersonnel, entre autres). Le phénomène de la circonlocution dépasse ainsi les seuls TC, qui s'insèrent alors dans un cadre plus général de rapport au latin : l'expression pluriverbale d'un seul contenu fonctionnel ou catégoriel.

\subsection{L'auxiliarité}

8 Le rôle des verbes être et avoir dans la conjugaison des langues vulgaires fait, très tôt, l'objet d'une innovation terminologique dans les grammaires du français ou rédigées dans cette langue. À partir de l'idée d'aide que ces verbes prêtent à la formation des temps, on forge le terme latin auxiliarium - la première attestation se trouve chez Pillot (1561 [1550] : 73) - ainsi que le français auxiliaire dans la décennie suivante. Le mot désigne une classe fonctionnelle ad hoc, le nouveau type venant s'ajouter aux types des verbes envisagées par la grammaire latine, en même temps qu'il sert à les distinguer: déjà, chez Pillot avoir était l'auxiliaire des actifs, comme être était celui des passifs (Colombat $2003: 103$ ). Dans les autres langues romanes, il faudra attendre le siècle suivant pour observer des attestations de termes équivalents. Toutefois, des remarques sur l'importance, pour le paradigme verbal, de ces verbes est attestée de façon générale dans toutes les traditions. Cependant, on ne saurait réduire le traitement de l'auxiliarité verbale à la seule notion d'aide (Chevalier 1999, Díaz Villalba 2017).

\subsection{La forme du verbe conjugué}

La question de la nature et des propriétés de la forme du verbe auxilié, id est le PTC, notamment en coalescence avec avoir, où cette dernière est presque toujours invariable, est un problème linguistique dont témoignent les premières descriptions. Les solutions à ce problème prennent des formes diverses : par exemple, le constat du caractère invariable de ces participes en espagnol (Miranda $1566: 214$, Alessandri 1560, f. 125v), les propositions qui mettent l'accent sur une propriété (ainsi le PTC, avec avere comme avec essere, a le trait sémantique " passé » chez Castelvetro (2004 [1563] : 297), alors que le même trait est absent dans le participe passif), ou encore les créations terminologiques (participe auxiliaire chez Dangeau 1927 [1694-1722]: 105), qui introduisent des subdivisions dans la classe du participe. L'exemple le plus poussé d'adaptation aux faits du vernaculaire apparaît chez Nebrija (1981 [1492] : 193), qui crée une nouvelle partie du discours, le nombre participial infinito (nom participial infini), se démarquant ainsi par son originalité de la tradition latine dont il hérite.

On peut remarquer que les réponses aux questions suscitées par les TC représentent des modes très différents d'appréhender les données : innovations terminologiques et traductions de termes latins (auxiliaire, rodeo), mobilisation de concepts empruntés aux figures de construction (périphrase), création de classes ad hoc (nombre participial infinito, verbe auxiliaire), ou encore ré-examen des propriétés d'une classe (le "participe passé " de Castelvetro). Parfois, une grammaire peut convoquer divers dispositifs. Ainsi, dans la méthode d'espagnol de Lancelot (1660a), on observe les trois solutions, ce qui tend à 
montrer qu'il s'agit de moyens de conceptualisation n'opérant pas sur le même plan. Dans certains textes, ils peuvent être opposés : Dumarsais (1751), qui soutient que les TC du français sont des périphrases, catégorise les PTC en tant que noms abstraits et métaphysiques ${ }^{1}$, en même temps qu'il critique le concept d'auxiliaire.

On met ici l'accent sur le fait que les deux dernières questions (c'est-à-dire le statut de l'auxiliaire, et celui de l'auxilié) sont à même de modifier le système de classification des mots de la grammaire latine, soit parce qu'elles introduisent des nouvelles sousclasses, soit parce qu'elles interrogent les propriétés des classes héritées. Certes, le concept de verbe auxiliaire connaitra un grand succès dans la tradition occidentale, ce qui pourrait nous faire oublier qu'une réflexion sur la nature et les propriétés de la forme de l'auxilié est présente dans la même tradition. Aussi proposerons-nous un panorama historique de la catégorisation de la forme du verbe principal, appelé communément participe, dans les TC de la voix active.

\section{Le participe, de la grammaire latine aux langues romanes}

12 Élaborée dans les grammaires grecques et latines antiques, la classe du participe dont hérite la tradition médiévale et moderne se présente comme une partie du discours, c'est-à-dire une classe au même titre que le pronom ou la préposition, par exemple. En effet, depuis la grammaire de Denys de Thrace (Tékhnē, chap. 15), le participe se caractérise par une nature hybride, associant des propriétés grammaticales du nom (l'adjectif est également compris dans cette classe) et du verbe. Ainsi, chez le latin Donat (IV ${ }^{\mathrm{e}} \mathrm{s}$.), le participe est défini en ces termes :

Participium est pars orationis, dicta quod partem capiat nominis partemque uerbi. Recipit enim a nomine genera et casus, a uerbo tempora et significationes, ab utroque numerum et figuram. Participiis accidunt sex, genus, casus, tempus, significatio, numerus, figura. (Donat, Ars maior, Holtz $1981: 644)^{2}$

13 Parmi les propriétés ou "accidents » attribués au participe, l'expression des valeurs temporelles (tempora) et des valeurs diathétiques (significationes), partagées avec le verbe, jouent un rôle important dans la classification des formes. Ainsi les formes en -tus du latin sont, chez Donat (Holtz 1981 : 644-645), identifiées comme des participes passifs (par exemple, amatus), des participes déponents (luctatus), ou des participes communs (criminatus) ${ }^{3}$. Quant au temps, ces formes sont des participes de proteritum (passé) chez Donat (ibid.), ainsi que chez Priscien (GL 2,557)4.

Les grammairiens latins observent différentes caractéristiques syntaxiques du participe, dont le régime, catégorie grammaticale consistant en un rapport de dépendance établi par le mot régissant sur le mot régi, qui adopte une forme casuelle spécifique. Si le régime ne fait pas partie des définitions antiques du participe, Priscien (GL 3.160) s'appuie, pour distinguer les participes des noms, sur l'idée que les mots qui suivent un participe se mettent au même cas que lorsqu'ils suivent un verbe. La construction du complément du participe à la manière d'un verbe appartient, tout comme les critères du temps et de la diathèse, à la boîte à outils dont disposent les grammairiens face aux problèmes de classification posés par certaines formes latines, même s'il ne s'agit pas des seuls critères employés (Visser 2010, Colombat 2019).

Dans les premières grammaires des vernaculaires, l'identification du participe de type aimé, équivalent du latin amatus se fait aisément. De même, les accidents sont hérités 
par les grammaires dans l'ensemble de la Romania (Díaz Villalba 2017). Parmi ceux empruntés au nom, le genre et le nombre permettent de traiter convenablement la morphologie, alors que le cas est écarté assez tôt. Toutefois, ce sont les accidents verbaux, la signification et le temps, qui servent à désigner les sous-classes du participe. Ainsi, dans les grammaires françaises des $\mathrm{XVI}^{\mathrm{e}}$ et $\mathrm{xVII}$ siècles, aimé est un participe " passif » et " passé » ou " prétérit » ${ }^{5}$, aimant est un participe « actif » et " présent ${ }^{6}$.

Du point de vue de son fonctionnement, le participe en latin peut être défini, dans une terminologie moderne, comme un mot flexionnel verbo-adjectif (parfois substantivé) : il remplit des rôles prototypiques d'adjectif (attribut ou modificateur adnominal, principalement), mais il peut aussi admettre des compléments propres au verbe. En revanche, le PTC associé à avoir, dans les langues romanes, est d'une autre nature. En effet, les TC sont le résultat d'un processus de grammaticalisation avancée, si bien que l'on ne peut plus parler d'adjectif pour la forme participiale. En revanche, cette dernière montre une forte coalescence avec le verbe auxiliaire (ordre fixe de deux constituants). De même, le rapport que le participe établissait, dans les langues médiévales, avec l'accusatif du verbe, manifesté par l'accord en genre et en nombre (du type les lettres que j'ai écrites), a disparu (espagnol, portugais) ou est resté cantonné à certaines configurations syntaxiques (français, italien), ce qui représente l'abandon d'une morphosyntaxe adjective du participe. Du point de vue sémantique, la perte de ce lien implique également l'absence de signification passive.

Bien évidemment, d'autres questions connexes doivent compléter la problématique (notamment la concurrence de l'auxiliaire être et la manifestation de l'accord du participe) ; cependant, nous nous occuperons uniquement de la catégorisation du PTC en coalescence avec avoir.

\section{Propositions de (re)catégorisation dans les grammaires}

18 La grammaire castillane de Nebrija élargit, nous l'avons souligné plus haut, la liste des parties du discours connues:

Una otra parte de la oración tiene nuestra lengua, la cual no se puede reduzir a ninguna de las otras nueve, $\tau$ menos la tiene el griego, el latín, ebraico $\tau$ arávigo. [...] E por que aún entre nos otros no tiene nombre, osemos la llamar nombre participial infinito: nombre, por que significa substancia $\tau$ no tiene tiempos; participial, por que es semejante al participio del tiempo passado; infinito, por que no tiene géneros, ni números, ni casos, ni personas determinadas. (Nebrija $1981: 193)^{7}$

19 Le grammairien fait ainsi preuve d'originalité, car il modifie la structure même du socle commun de la tradition occidentale. Cependant, il ne sera pas suivi. Les grammairiens de la série se contentent souvent d'introduire des sous-classes du participe ad hoc, comme Irson (1656:19-20) qui forge le participe commun actif: commun parce que la même forme présente des valeurs différentes, en l'occurrence, le même participe exprime la passivité dans d'autres constructions. Outre ces innovations, les textes attestent également la recatégorisation dans d'autres sous-classes du verbe. C'est ainsi que Figueiredo conçoit le PTC comme un supin :

o Supino, que he a voz do Infinito Activa, que se ajunta em todos os Verbos aos dous Auxiliares Ter, e Haver para formar com elles os Tempos compostos, ou de circumloquio, nem tẽ plural, nem terminação feminina, e nisto se distingue dos Participios do Preterito 
Passivos, os quaes, aindaque por elle se formem, tem todos ambos os generos, e terminações, por ser isto da natureza do Participio. (Figueiredo 1811 [1799] : 84-85) ${ }^{8}$ du PTC dans l'ensemble des grammaires des langues romanes fait émerger la récurrence significative des options envisagées. Le tableau 1 réunit 42 ouvrages, rangés par ordre de leur première édition. La limite chronologique de 1799 est arbitraire, la série pouvant s'étendre au XIX siècle, notamment chez les auteurs des grammaires générales. Néanmoins, nous analysons les trois premiers siècles de grammatisation, qui correspondent à l'élaboration de la problématique.

Afin de saisir les contours précis de la construction historiographique que dessine le tableau, deux remarques s'imposent. D'une part, nous ne saurions trop insister sur le fait que les désignations ne laissent pas toujours deviner le choix de la catégorisation. À titre d'exemple, Castelvetro (1563) emploie partefice passato (participe passé), appellation traditionnelle, pour parler des seuls PTC, porteurs de l'expression du temps, contrairement aux autres participes, dépourvus, aux yeux de l'auteur, d'une telle propriété. D'autre part, il n'y a pas homogénéité, à l'intérieur de la série, dans la façon de catégoriser, ni par la portée théorique, ni par le dispositif descriptif, ni non plus par l'organisation des classes de mots. En effet, le traitement reste parfois peu approfondi : chez Mambelli (1685: 547), par exemple, on constate simplement l'idée que le participe prétérit possède un sens actif lorsqu'il est régi par le verbe avere (avoir), alors que sa sémantique est passive en coalescence avec le verbe essere (être). En revanche, nombreux sont les grammairiens (Nebrija 1492, Meigret 1550, Ramus 1562, Arnauld et Lancelot 1660, Lancelot 1660, pour ne nommer qu'eux) qui accordent une place prépondérante à la théorisation sur ce type de mots. Néanmoins, les enjeux sont sans commune mesure. Ainsi, Nebrija tente de se démarquer par son originalité de la tradition latine dont il hérite (Tollis 1998). Dans le même sens, Meigret, veut poser des bases rationnelles pour une description du français reposant sur les données propres à cette langue. Ramus, quant à lui, refonde le système des parties du discours selon un principe binaire, s'écartant ainsi des classifications traditionnelles. C'est ainsi que le grammairien oppose les mots exprimant le nombre (le nom et le verbe) et les autres mots, les mots "avec nombre " pouvant être à leur tour divisés en mots de nombre fini, tels que les formes conjuguées du verbe, ou en mots de nombre infini, comme le gérondif ou l'infinitif, dont le PTC est une sous-classe. La démarche de PortRoyal, quant à elle, explore la possibilité d'une catégorisation générale des participes des langues modernes, dont les faits du français, de l'espagnol et de l'italien sont, aux yeux de ces grammairiens, des manifestations concrètes des mêmes principes. En somme, les modalités des traitements, les arguments et les enjeux épistémologiques sont particuliers (Fournier 2011, Díaz Villalba 2012). Toutefois, c'est la mise en série d'un grand nombre de textes qui nous permet d'observer la cristallisation d'une problématique générale stable dans la durée. 
Tableau 1. Catégorisation du participe des temps composés (1492-1799).

\begin{tabular}{|c|c|c|c|c|}
\hline date & $\begin{array}{l}\text { métalangue/langue- } \\
\text { objet (si différente) }\end{array}$ & auteur & \begin{tabular}{|l} 
désignation pour le \\
participe des temps \\
composés
\end{tabular} & $\begin{array}{ll}\text { choix } & \mathrm{de} \\
\text { l'étiquette } & \end{array}$ \\
\hline 1492 & ES & Nebrija & nombre participial infinito & nom + participe \\
\hline 1530 & AN/FR & Palsgrave & participle preterit & participe \\
\hline 1550 & FR & Meigret & $\begin{array}{l}\text { infinitif passé et actif, } \\
\text { infinitif prétérit }\end{array}$ & infinitif \\
\hline 1563 & IT & Castelvetro & partefice passato & participe \\
\hline 1562 & FR & Ramus & infiny (ou perpetuel) preterit & infinitif \\
\hline 1570 & LA/FR & Cauchie & vox proteriti & forme verbale \\
\hline 1607 & FR & Maupas & participe commun & participe \\
\hline 1619 & PT & Roboredo & gerundio de preterito & gérondif \\
\hline 1627 & ES & Correas & partizipio ministro & participe \\
\hline 1651 & ES & Villar & supino & supin \\
\hline 1656 & FR & Irson & participe commun actif & participe \\
\hline 1660 & FR & $\begin{array}{l}\text { Arnauld et } \\
\text { Lancelot }\end{array}$ & gérondif & gérondif \\
\hline 1660 & FR/IT & Lancelot & gérondif & gérondif \\
\hline 1660 & FR/ES & Lancelot & gérondif & gérondif \\
\hline 1675 & FR & Bouhours & supin & supin \\
\hline 1681 & FR & Vairasse d'Allais & $\begin{array}{l}\text { participe (de signification } \\
\text { active) }\end{array}$ & participe \\
\hline 1685 & IT & Mambelli & $\begin{array}{l}\text { participio preterito de } \\
\text { significazione attiva }\end{array}$ & participe \\
\hline 1694 & FR & Dangeau & participe auxiliaire & participe \\
\hline 1705 & FR & $\begin{array}{l}\text { Régnier- } \\
\text { Desmarais }\end{array}$ & $\begin{array}{l}\text { participe absolu, participe } \\
\text { passé adjectif \& actif }\end{array}$ & participe \\
\hline 1708 & FR/ES & Vayrac & participe actif & participe \\
\hline 1719 & FR & Vallange & supin & supin \\
\hline
\end{tabular}




\begin{tabular}{|c|c|c|c|c|}
\hline 1728 & FR/ES & Torre y Ocón & participe prétérit actif & participe \\
\hline $\begin{array}{l}1730 \\
1751\end{array}$ & FR & Dumarsais & $\begin{array}{l}\text { supin, nom substantif } \\
\text { abstrait \& méthaphysique }\end{array}$ & supin, nom \\
\hline 1730 & FR & Restaut & $\begin{array}{l}\text { participe passif } \quad \text { (de } \\
\text { signification active) }\end{array}$ & participe \\
\hline 1744 & FR & Vallart & participe auxiliaire & participe \\
\hline 1754 & FR & Duclos & supin & supin \\
\hline 1760 & FR & D’Açarq & supin & supin \\
\hline $\begin{array}{l}1765 a, b \\
1767\end{array}$ & FR & Beauzée & supin & supin \\
\hline 1769 & ES & San Pedro & $\begin{array}{l}\text { participio de preterito } \\
\text { (activo) }\end{array}$ & participe \\
\hline 1770 & PT & Lobato & $\begin{array}{l}\text { participio de preterito } \\
\text { indeclinavel }\end{array}$ & participe \\
\hline 1770 & ES & Puig & $\begin{array}{l}\text { participio de preterito } \\
\text { (significacion activa, voz } \\
\text { neutra) }\end{array}$ & participe \\
\hline 1771 & IT & Soave & $\begin{array}{l}\text { participio passato + nome } \\
\text { universale oggetto }\end{array}$ & $\begin{array}{l}\text { participe }+ \text { nom } \\
\text { sous-entendu }\end{array}$ \\
\hline 1771 & ES & $\begin{array}{l}\text { Real Academia } \\
\text { Española (RAE) }\end{array}$ & participio auxîliar & participe \\
\hline 1775 & FR & Condillac & participe substantif & participe \\
\hline 1783 & PT & Bacelar & nominativo indeclinavel & nom \\
\hline 1793 & ES & Muñoz & $\begin{array}{l}\text { terminacion neutra del } \\
\text { participio de pretérito } \\
\text { perfecto }\end{array}$ & participe \\
\hline 1796 & ES & Ballot & $\begin{array}{l}\text { participio de pretérito (de } \\
\text { significacion activa) }\end{array}$ & participe \\
\hline 1798 & FR & Sicard & supin & supin \\
\hline 1799 & FR & Serreau & participe absolu & participe \\
\hline 1799 & PT & Figueiredo & supino & supin \\
\hline
\end{tabular}

AN : anglais, ES : espagnol, FR : français, IT : italien, LA : latin, PT : portugais 


\subsection{Les axes du traitement} forme des tiroirs verbaux, ce qui écarte incontestablement ces formes du comportement des autres participes. Six auteurs mettent en avant cette propriété en inscrivant l'auxiliarité dans les désignations ${ }^{10}$. Maupas (1618, f. 97r) affirme que le participe est commun parce qu'il sert à former les temps actifs comme les passifs, se joignant aux deux auxiliaires être et avoir. D'autres auteurs vont plus loin : le choix de participe auxiliaire (Dangeau, op. cit.) ou de participio auxîliar (RAE 1771 : 182), ainsi que de partizipio ministro (corrélat de verbos ministrales, i.e. verbes auxiliaires, chez Correas (1984 [1627], 157 sq), représente un transfert d'une innovation terminologique (verbes auxiliaires) de la classe du verbe vers la classe du participe. L'approche adoptée par ces grammairiens met l'accent sur le caractère complémentaire de la relation auxiliaireparticipe. En effet, Correas justifie la désignation par le fait que le participe en question n'apparaît jamais seul, mais « en compañia de los tienpos que aiuda á formar i suplir » (op. cit., p. 165) (en compagnie des temps qu'il aide à former et auxquels il supplée), dans une formulation assez proche de la définition d'un verbe auxiliaire. Dangeau (1927 [1694-1722] : 100-105) et Vallart (1744: 234), de leur côté, préfèrent le terme d'usage pour parler de l'essence fonctionnelle du participe auxiliaire, là où RAE (1771: 182) qualifie d'oficio (office) le rôle de la classe.

Le deuxième procédé consiste à trouver, pour ces formes, une classe de la grammaire latine permettant d'expliquer leurs caractéristiques linguistiques. Les options privilégiées correspondent aux formes non finies du verbe : le gérondif, l'infinitif et le 
supin. Ce phénomène illustre une conséquence de la latinisation de la grammaire des vernaculaires. On peut être sensible au fait que les trois possibilités catégorielles offertes par le schéma théorique et descriptif (les classes verbales que connaît la grammaire latine sont précisément, en dehors des formes personnelles, au nombre de trois) sont envisagées dans les grammaires de différentes traditions, comme si le modèle tendait à produire, de manière prévisible, des réflexions de même nature pour traiter certaines données.

Or, chacune des options représente des enjeux différents et pose des problèmes spécifiques. L'infinitif constitue, dans la tradition occidentale, un mode fonctionnant, en réalité, comme un chapeau pour l'ensemble des formes verbo-nominales. Cette essence générale a dû conduire Meigret au choix terminologique d'infinitif prétérit pour le PTC, mais il n'y a pas de coïncidence entre les propriétés de cette sous-classe et celles de l'autre infinitif (aimer), qui ne saurait, par exemple, exprimer une quelconque valeur temporelle. L'infinitif de Ramus, sous le terme de perpétuel ou d'infini (1562: 52), est, quant à lui, une subdivision primaire des verbes (opposable à l'ensemble des formes finies), dont les espèces sont l'infinitif présent, le gérondif et l'infini prétérit (le PTC), toutes étant dépourvues de l'expression formelle du nombre. Les approches théoriques de Meigret et de Ramus n'ont pas eu de successeurs directs. Si Cauchie (1586, f. 38 r) se fait l'écho des débats sur la catégorisation du PTC en tant qu'infinitif, il n'adhère pas à ce choix. Toutefois, la possibilité théorique restant disponible, des textes bien plus récents (Noboa 1839, Jullien 1849) optent pour la catégorisation du PTC en infinitif.

Le choix du gérondif est également circonscrit dans le temps (Roboredo 1619:18, 33, Arnauld et Lancelot $1660: 133 \mathrm{sv}$, Lancelot 1660a: 74, 1660b: $87 \mathrm{sv}$ ). Il convient de rappeler que, dans la tradition française au $\mathrm{XVI}^{\mathrm{e}}$ siècle, le terme de gérondif s'identifie exclusivement à des formes latines et à leurs traductions en langue vernaculaire, et pas encore à des formes françaises en -ant. En effet, la catégorisation du gérondif repose sur la classe du gerundium latin (type amandum, $-i,-o$ ), c'est-à-dire, une forme nominale du verbe sans variation de genre et de nombre; il s'agit, en quelque sorte, d'une déclinaison de l'infinitif. Cette conception se retrouve encore, en grande partie, au siècle suivant. Néanmoins, les auteurs de Port-Royal élèvent le gérondif au rang de catégorie générale et abstraite dont ils ne retiennent que les éléments définitoires ( $c f$. infra), ce qui leur permet de percevoir des "gérondifs» dans les PTC des langues modernes (le français dans la Grammaire générale et raisonnée (GGR), mais aussi l'italien et l'espagnol dans les méthodes d'apprentissage de Lancelot). Ainsi, le participe invariable est un gérondif qui régit l'accusatif dans « io ho riceuuto la vostra lettera, j'ay receu vostre lettre " (Lancelot 1660b: 90), alors que le participe qui montre l'accord n'est pas un mot régissant, par exemple « la lettera che voi mi hauete mandata, la lettre que vous m'auez enuoyée » (ibid.).

Cependant, la théorie d'une classe appelée gérondif pour les TC n'a pas eu la chance d'être entérinée, malgré le rôle historique de la GGR dans la tradition grammaticale. Régnier-Desmarais (1706: 486 sq.) fait une critique de la catégorisation de Port-Royal, en établissant une distinction nette entre les participes et les vrais gérondifs. En outre, le terme s'étant spécialisé dans les grammaires du XVII siècle pour nommer les formes françaises du type (en) aimant, une éventuelle reprise de cette étiquette pour les PTC aurait sans doute brouillé les distinctions des classes grammaticales. groupe de textes bien plus étoffé. Le rapprochement du participe passif et du supin 
s'explique aisément en raison du morphème homonyme de ces classes en latin, le participe passif au neutre ayant une forme identique neutre au nominatif/accusatif (v.gr. amatum). La ressemblance se prête à des métaphrases des exemples vernaculaires à l'aide du latin. Par exemple, Bouhours exploite une telle possibilité dans l'extrait suivant :

Avec le verbe avoir il [le participe] est naturellement indéclinable, n'ayant ni genre, ni nombre. J'ay receû vos Lettres; j'ay reçeû vos Livres, parce que c'est plûtost le supin des Latins, que le participe ; \& que c'est comme si on disoit, habeo acceptum Litteras, habeo acceptum libros. (Bouhours $1675: 360$ )

L'avantage de la similitude formelle est décisive: l'existence de cette homonymie morphématique en latin, modèle de toute description grammaticale, est une raison solide pour postuler l'existence de deux mots aimé, l'un variable, l'autre invariable.

Beauzée approfondit cette idée en l'étendant, car l'auteur développe dans deux articles de l'Encyclopédie (Beauzée 1765a, 1765b) avec force arguments, la thèse selon laquelle le PTC doit être considéré comme un supin (Díaz Villalba 2015a), dans plusieurs langues modernes (allemand, espagnol, français et italien), lorsqu'il est invariable, tandis que les formes participales qui manifestent l'accord dans les lettres que mon père a reçues (1765a : 96) sont catégorisées comme des participes. Il est question, dans ces textes, d'une catégorisation plus abstraite et générale sur la base d'une poignée de propriétés grammaticales comparables. Mais ce type d'approche n'est pas l'apanage des auteurs des grammaires générales, il est représenté depuis la Renaissance. Ainsi, le traitement du PTC à l'aide d'une des trois classes verbo-nominales réside dans une série de caractéristiques :

a) sous l'angle de la morphologie, les classes précitées (infinitif, gérondif, supin) partagent l'absence d'une variation formelle en genre et en nombre, contrairement aux participes passés, qui, eux, sont variables (cf. supra Bouhours);

b) sous l'angle de la sémantique, on attribue à ces formes verbales la diathèse active, tandis que les participes passés sont passifs.

[...] el participio simple, que algunas vezes llamamos supino [...] en los circunloquios de la voz activa significa accion, $y$ assi dezir yo e amado, es dezir, yo e sido de quien procedio la accion de amar. (Villar 1651, p. 79) ${ }^{11}$

c) sous l'angle de la construction, la nature active présente un corrélat syntaxique, le régime, dans la mesure où ces formes manifestent la capacité de régir le même cas que les formes personnelles ou conjuguées du verbe à la voix active (cela est aussi vrai pour les participes actifs). Les grammairiens de Port-Royal s'appuient notamment sur ce critère dans le traitement de la classe :

Ainsi pour resoudre la difficulté proposée, je dis que dans ces façons de parler, j'ay aimé la chasse, j'ay aimé les livres, j'ay aimé les sciences, la raison pourquoy on ne dit point ; j'ay aimée la chasse, j'ay aimez les livres ; c'est qu'alors le mot aimé ayant le regime du verbe, est gerondif, et n'a point de genre ny de nombre. (Arnauld et Lancelot $1660: 133)$

Les qualités grammaticales évoquées ci-dessus sont, par ailleurs, présentes chez d'autres grammairiens du corpus, dans des désignations du type "participe actif » ou "participe indéclinable» en tant que spécifications supplémentaires, alors que ces traits grammaticaux sont intrinsèques aux classes de l'infinitif, du gérondif et du supin, ce qui constitue un argument de poids pour les grammairiens de la période. On peut comprendre que les trois étiquettes de la grammaire latine représentent de «bonnes candidates " pour la recatégorisation des formes qui nous intéressent. 

classes en tant qu'elles sont conceptualisées comme classes proches du substantif. En effet, les grammairiens assimilent ces classes verbales à des noms. Ainsi, Lancelot affirme à propos de l'infinitif : «L'Infinitif venant à perdre l'affirmation qui est propre au verbe, doit estre consideré comme un nom substantif dans toutes les langues.» (Lancelot 1660b : 82).

C'est pourquoi la prise en compte des seules désignations peut s'avérer insuffisante pour saisir tous les enjeux de la catégorisation. Ce phénomène est patent dans le caractère nominal indissociable des formes non personnelles du verbe. Par ailleurs, la dimension de parenté avec le nom entre en résonance avec d'autres options théoriques, telles que nombre participial infinito (Nebrija 1492), nom substantif abstrait et métaphysique (Dumarsais 1730) participe substantif (Condillac 1775), ou encore nominativo ${ }^{12}$ indeclinavel (Bacelar 1783). La mise en réseau des textes fait ressortir le fait que les connexions possibles entre les propositions théoriques sont multiples et complexes.

\subsection{La polycatégorisation dans le traitement des questions particulières}

Un panorama très général encourt le risque de réduire, en quelque sorte, l'épaisseur historique de l'objet. Il en va ainsi de la manière dont la problématique s'inscrit dans l'histoire de la description de faits de langues particuliers.

S'agissant de la tradition française, la polycatégorisation s'insère dans l'histoire de la règle de l'accord du participe passé, en cours d'élaboration en français classique (N. Fournier $1998: 315-329)$. Dans cette tradition, les choix théoriques présentés dans notre étude concernent quasi exclusivement le type avoir + participe invariable (par exemple dans la citation de Lancelot reproduite plus haut), au détriment des autres configurations, qui comportent, aux yeux de certains grammairiens, le véritable participe. En ce sens, la problématique de l'accord est investie d'une réflexion approfondie sur la nature des mots et leurs rapports sémantiques et syntaxiques. Cela explique certainement la forte présence d'auteurs français dans notre série de recatégorisations : les débats sur la règle de l'accord ont fourni, dans l'histoire de la grammaire française, un terrain favorable à la réflexion syntaxique (Fournier 2011).

41 Le problème de la nature du participe est également remarquable dans les grammaires de l'espagnol et du portugais. L'accord du participe dans la périphrase avec tener a conduit, d'abord dans les grammaires espagnoles parues en France, ensuite en Espagne, à établir des divisions parmi les verbes auxiliaires de l'espagnol (tener et haber pour l'actif, ser et estar pour le passif), en corrélation avec une polycatégorisation des participes selon qu'ils s'accordent ou non (Díaz Villalba 2015b). On peut ajouter que, dans la problématique des doubles participes (réguliers et irréguliers) de l'espagnol et du portugais, la règle de distribution qui commence à être élaborée dans le dernier tiers du XVIII ${ }^{e}$ siècle, n'est pas étrangère aux distinctions catégorielles : l'existence de deux formes tend à confirmer que ces participes sont de natures différentes. Ainsi, Figueiredo (1811 [1799]: 91-92) établit deux classes distinctes pour les participes doubles portugais : le supin se joint aux auxiliaires ter et haver, le participe se joint à l'auxiliaire ser.

Histoire Épistémologie Langage, 42-1 | 2020 


\section{Conclusion}

La catégorisation des mots, dont la macrostructure visible est le système des parties du discours, constitue un dispositif essentiel de la grammatisation des vernaculaires romans. Notre étude s'attache à examiner la façon dont les grammairiens adoptent ce cadre théorique et explicatif pour traiter de nouveaux sujets.

La mise en série d'une quarantaine de grammaires, rarement réunies dans l'analyse, est guidée par la présence d'un même objet: tous les textes proposent un terme catégorisant, souvent soutenu par une armature théorique, pour conceptualiser la nature ou les propriétés du participe des TC. Il nous est apparu, dans les textes sélectionnés, deux grands choix dans la façon de s'approprier les outils de la catégorisation. Le premier consiste à modifier le système de classification, soit par l'invention d'une partie du discours (l'option de Nebrija: le nombre participial infinito), soit par l'introduction de nouvelles subdivisions au sein d'une classe, telles le partizipio ministro (Correas). Généralement, le traitement de la sous-classe représente la mise en avant d'une propriété remarquable : partefice passato (Castelvetro) ou terminacion neutra (Muñoz). Le deuxième choix met à contribution des classes grammaticales (nom, gérondif, infinitif, supin) qui présentent des caractéristiques aptes à expliquer le comportement du participe. La recatégorisation du PTC à l'aide de ces étiquettes n'est d'ailleurs pas incompatible avec le premier choix. Ainsi, l'infinitif passé actif (Meigret) n'est pas une simple transposition de l'infinitif latin, mais le texte du grammairien implique une ré-élaboration argumentée de la classe pour expliquer les faits linguistiques du français. C'est pourquoi, les deux options constituent des possibilités complémentaires des ressources disponibles dans la boîte à outils qu'est le modèle fondé en classes et en accidents (propriétés ou catégories grammaticales).

Optant pour une approche d'ensemble ou extensive, l'investigation doit être complétée par des analyses particulières du traitement dans des parcelles de la série. Choisir le long terme en histoire, c'est aussi se donner les moyens de mieux comprendre la transmission des choix explicatifs, ainsi que le rythme du changement théorique. Sans doute l'existence d'une communauté du savoir métalinguistique, issue du transfert du cadre latin, a-t-elle favorisé la circulation des idées dont témoigne la diffusion de certaines propositions théoriques.

Néanmoins, l'objectif de la présente étude se veut plus général: il s'agit d'une illustration de l'intérêt de l'histoire comparée des grammaires. Ainsi, l'alignement d'un ensemble de textes permet de délimiter un objet historiographique dans la grammatisation des langues romanes, dont on peut affirmer la longévité et la récurrence.

BIBLIOGRAPHIE 


\section{Sources primaires}

Alessandri, Giovanni M. 1560. Il Paragone della lingua toscana et castigliana. Naples : Cancer.

Anonyme. 1966 [1559]. Gramática de la lengua vulgar de España, édition établie par R. de Balbín et A. Roldán. Madrid : CSIC.

Arnauld, Antoine \& Claude Lancelot. 1660. Grammaire générale et raisonnée. Paris : Le Petit.

Bacelar, Bernardo de Lima e Melo. 1996 [ 1783]. Gramática Filosófica da Língua Portuguesa, facsimilé, éd. établie par Amadeu Torres. Lisbonne : Academia Portuguesa da História.

Ballot, Joseph P. 1796. Gramática de la lengua castellana. Barcelone : Piferrer.

Barros, João. 1540. Grammatica da lingua portuguesa. Lisbonne : Rodrigues.

Beauzée Nicolas. 1765a. Participe. Encyclopédie ou Dictionnaire raisonné des sciences, des arts et des métiers dir. par Denis Diderot \& Jean Le Rond d'Alembert, vol. 12, 90-99. Paris : Briasson, David, Le Breton et Durand.

Beauzée Nicolas. 1765b. Supin. Encyclopédie ou Dictionnaire raisonné des sciences, des arts et des métiers dir. par Denis Diderot \& Jean Le Rond d'Alembert, vol. 15, 670-671. Paris : Briasson, David, Le Breton et Durand.

Beauzée Nicolas. 1767. Grammaire Générale, 2 vol. Paris : Barbou.

Bouhours, Dominique. 1675. Remarques nouvelles sur la langue françoise. Paris : Mabre-Cramoisy. Castelvetro, Lodovico. 2004 [1563]. Giunta fatta al ragionamento degli articoli et de' verbi di Messer Pietro Bembo, éd. établie par Matteo Motolese. Roma/Padova : Antenore.

Cauchie, Antoine. 1586 [1570]. Grammaticae gallicae libri tres. Strasbourg : Jobin.

Condillac, Étienne Bonnot de. 1775. Grammaire (Cours d'étude pour l'instruction du Prince de Parme, vol. I). Parme : Imprimerie Royale.

Correas, Gonzalo. 1984 [1627]. Arte Kastellana, éd. établie par Manuel Taboada Cid. Universidad de Santiago de Compostela.

D’Açarq, Jean-Pierre. 1760. Grammaire française philosophique, 2 vol. Genève : Moreau \& Lambert.

Dangeau, Abbé Louis de Courcillon de. 1927 [1694-1722]. Opuscules sur la grammaire, éd. établie par Manne Ekman. Uppsala : Almquist \& Wiksells.

Denys, le Thrace. 1998. La grammaire de Denys le Thrace, $2^{e}$ éd., texte établi et traduit par Jean Lallot. Paris : CNRS Editions.

Duclos, Charles. 1754. Remarques. Grammaire générale et raisonnée par Antoine Arnauld \& Claude Lancelot. Paris : Prault.

Dumarsais, César Chesneau. 1987 [1729-1756]. Les véritables principes de la grammaire et autres textes, éd. établie par Françoise Douay-Soublin. Paris : Fayard.

Dumarsais, César Chesneau. 1751. Auxiliaires. Encyclopédie ou Dictionnaire raisonné des sciences, des arts et des métiers dir. par Denis Diderot \& Jean Le Rond d'Alembert, vol. 1, 903-904. Paris : Briasson, David, Le Breton et Durand.

Estienne, Robert. 1557. Traicté de la grammaire Francoise. Genève : Estienne.

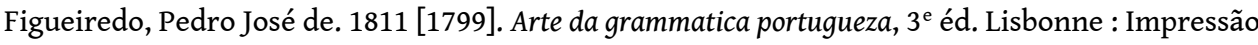
Régia. 
Irson, Claude. 1656. Nouvelle methode pour apprendre facilement les principes et la pureté de la langue françoise contenant plusieurs traitez. Paris : Meturas.

Jullien, Bernard. 1849. Cours supérieur de grammaire. Paris : Hachette et Cie.

Lancelot, Claude. 1660a. Nouvelle Methode pour apprendre facilement et en peu de temps la langue espagnole. Paris : Le Petit.

Lancelot, Claude. 1660b. Nouvelle Methode pour apprendre facilement et en peu de temps la langue italienne. Paris : Le Petit.

Lobato, António J. dos Reis. 1770. Arte da grammatica da lingua portuguesa. Lisboa : Regia Officina Typographica.

Mambelli, Marcantonio. 1685. Osservazioni della lingua italiana. Forlì : Selva.

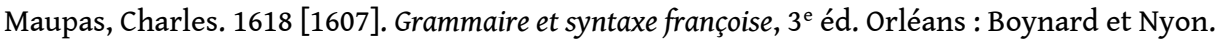

Meigret, Louis. 1980 [1550]. Le traité de la grammaire française, éd. établie par Franz-Josef Hausmann. Tübingen, Gunter Narr.

Miranda, Giovanni. 1566. Osservationi della lingua castigliana. Venise : de' Ferrari.

Muñoz Álvarez, Agustín. 1799 [1793]. Gramática de la lengua castellana. Séville : de la Puerta.

Nebrija, Elio Antonio de. 1981 [1492]. Gramática de la lengua castellana, éd. établie par Antonio Quilis. Madrid : Editora Nacional.

Noboa, Andrés M. de. 1839. Nueva gramática de la lengua castellana segun los principios de la filosofía gramatical. Madrid : Aguado.

Palsgrave, John. 1852 [1530]. L'éclaircissement de la langue française. Paris : Imprimerie Nationale.

Pillot, Jean. 1561 [1550]. Gallicee linguce institutio, latino sermone conscripta. Paris : Etienne Grouleau.

Priscien. 1855-1859. Institutionum grammaticorum libri XVII, vol. 2-3, éd. établie par Heinrich Keil. Leipzig : Teubner.

Puig, Salvador. 1770. Rudimentos de la Gramatica Castellana. Barcelone : Piferrer.

Ramus, Petrus. 1562. GramerĘ. Paris : Wechel.

Real Academia Española. 1984 [1771]. Gramática de la lengua castellana. Madrid : Editora Nacional. Fac-similé, étude de Ramón Sarmiento.

Régnier-Desmarais, François Séraphin. 1706 [1705]. Traité de la grammaire françoise. Paris : Coignard.

Restaut Pierre. 1758 [1730]. Principes généraux et raisonnés de la Grammaire françoise. Paris : Vve Lottin, Desaint et Saillant.

Roboredo, Amaro de. 2002 [1619]. Methodo Grammatical para todas as Linguas, éd. établie par Marina A. Kossarik. Lisbonne : Imprensa Nacional-Casa da Moeda.

San Pedro, Benito de. 1769. Arte del romance castellano. Valence : Monfort.

Serreau, Jean-Edme. 1799 (floréal an VII). Grammaire raisonnée, ou principes de la langue française, appropriés au génie de la Langue. Paris : Richard, Caille et Ravier.

Sicard, Roch-Ambroise-Cucurron Abbé. 1808 [1798]. Elémens de grammaire générale, appliqués à la langue française, $3^{\mathrm{e}}$ éd. Paris : Deterville. 
Soave, Francesco. 2001 [1771]. Gramatica ragionata della lingua toscana, éd. établie par S. Fornara. Pescara : Università Editrice.

Torre y Ocón, Francisco de la. 1728. Nuevo methodo breve, util y necessario para aprender a escribir entender y pronunciar las dos principales lenguas española y francesa. Madrid : Aritzia.

Vairasse d'Allais, Denis. 1681. Grammaire méthodique. Paris : chez l'auteur.

Vallange, de. 1721 [1719]. Grammaire françoise raisonée. Paris : Jombert, et al.

Vallart, Abbé Joseph. 1744. Grammaire Françoise. Paris : Desaint et Saillant.

Vayrac, abbé de. 1708. Nouvelle grammaire espagnole. Paris : s. n.

Villar, Juan de. 1651. Arte de la lengua española. Valence : Verengel.

\section{Sources secondaires}

Chevalier, Jean-Claude. 1999. La notion d'auxiliaire verbal. Origine et développement. Langages $135: 22-32$

Colombat, Bernard. 2003. Institution de la langue française, Jean Pillot, Gallicae linguae institutio, 1561. Paris : Honoré Champion.

Colombat, Bernard. 2019. Participium. Histoire des parties du discours. Dir. par Bernard Colombat \& Aimée Lahaussois (Orbis Supplementa, 46.), 319-326. Louvain : Peters.

Díaz Villalba, Alejandro. 2012. Le participe dans les grammaires des langues romanes, de la Renaissance à la grammaire générale (1492-1660). Dans Vers une histoire générale de la grammaire française. Dir. par Bernard Colombat, et al., 653-670. Paris : Honoré Champion.

Díaz Villalba, Alejandro. 2015a. La catégorie « supin » dans l'Encyclopédie de Diderot et D’Alembert. Dans Les classifications en linguistique : problèmes, méthodologie, enjeux. Dir. par Angelina Aleksandrova, et al., 125-143. Bochum : Westdeutscher Universitätsverlag.

Díaz Villalba, Alejandro. 2015b. La classe des verbes auxiliaires dans les grammaires de l'espagnol en France au XVII ${ }^{\mathrm{e}}$ siècle. Dans La terminología gramatical del español y del francés : emergencias y transposiciones, traducciones y contextualizaciones. Dir. par Cécile Bruley \& Javier Suso López, 97-119. Franfurt am Main : Peter Lang.

Díaz Villalba, Alejandro. 2017. Le participe dans les grammaires des langues romanes ( $\mathrm{XV}{ }^{e}$-XVIII ${ }^{e}$ siècles). Histoire comparée d'une classe grammaticale. Thèse de doctorat. Université Sorbonne Nouvelle et Universidad de Salamanca.

Fournier, Jean-Marie. 2011. À propos des règles dans les grammaires françaises de l'âge classique : forme, fonction, statut (le cas de l'accord du participe passé). Dans History of Linguistics 2008, Selected Papers from the Eleventh International Conference on the History of the Language Sciences. Dir. par Gerda Hassler, 265-276. Amsterdam/Philadelphia : Benjamins.

Fournier, Jean-Marie \& Raby, Valérie. 2014. Retour sur la grammatisation : l'extension de la grammaire latine et la description des langues vulgaires. Dans Penser l'histoire des savoirs linguistiques. Hommage à Sylvain Auroux. Dir. par Sylvie Archaimbault, et al. 337-350. Lyon : ENS Éditions.

Fournier, Nathalie. 1998. Grammaire du français classique. Paris : Belin. 
Fournier, Nathalie, Samain, Didier \& Lahaussois, Aimée. 2019. Article. Dans Histoire des parties du discours. Dir. par Bernard Colombat \& Aimée Lahaussois (Orbis Supplementa, 46.), 165-201. Louvain : Peters.

Gómez Asencio, José. 2015. Terminología gramatical española de los principios/Principios de la terminología gramatical española. Dans La terminología gramatical del español y del francés. Emergencias y transposiciones, traducciones y contextualizaciones. Dir. par Cécile Bruley \& Javier Suso López, 25-74. Franfurt am Main : Peter Lang.

Haßler, Gerda. 2000. Les séries de textes dans l'histoire de la linguistique. Dans Actes du XII ${ }^{e}$ Congrès International de Linguistique et Philologie Romanes. Dir. par Annick Anglebert, et al., vol. 1, 97-102. Tübingen : Niemeyer.

Holtz, Louis. 1981. Donat et la tradition de l'enseignement grammatical. Étude sur l'Ars Donati et sa diffusion (IV $-I X^{e}$ siècle) et édition critique. Paris : CNRS.

Tollis, Francis. 1998. À propos des circunloquios du verbe castillan chez Nebrija. Le nombre participial infinito. La description du castillan au Xve siècle. Villena et Nebrija par Francis Tollis, 91-121. Paris : L'Harmattan.

Visser, Louise. 2010. The Participle in Latin Grammars in the Early Middle Ages (400-900 AD). A Linguistic, Philological, and Cultural-historic Study. Thèse de doctorat. Katholike Universiteit Leuven, Louvain.

\section{NOTES}

1. Le terme métaphysique joue un rôle important dans le métalangage grammatical de Dumarsais (Seguin 1980), dont l'emploi le plus connu se trouve dans adjectif métaphysique, catégorisation qu'approfondira Beauzée (Fournier et al., 2019 : 177-178) pour traiter ce qu'on appelle aujourd'hui les déterminants. La désignation noms abstraits et métaphysiques renvoie à une conceptualisation propre au langage de l'ordre du contenu grammatical; ces «noms » se distinguent, en vertu de leur signification méta-abstraite, par exemple dans avoir aimé, des autres noms abstraits (honte, froid, soif), qui ont une signification lexicale, par exemple, dans avoir honte (Díaz Villalba 2015a).

2. «Le participe est une partie du discours ainsi appelée du fait qu'elle prend une part du nom et une part du verbe. En effet, il prend du nom les genres et les cas, du verbe les temps et les significations; il prend de l'un et de l'autre le nombre et la figure. Six accidents échoient au participe : le genre, le cas, le temps, la signification, le nombre et la figure. » (Nous traduisons)

3. On rappellera que les verbes communs sont, chez Donat (Holtz 1981 : 635-636), ceux terminés en $-r$ à la première personne qui peuvent se construire à l'actif ou au passif : criminor te «je t'accuse », face à criminor a te «je suis accusé par toi». Les classifications des verbes selon la diathèse articulent, dans les grammaires latines, des critères formels et sémantiques, pour arriver à différentes divisions du verbe : actif, passif, neutre, déponent et commun.

4. Chez Priscien, le participe en -tus exprime sous une seule forme les valeurs de prétérit parfait et de plus-que-parfait.

5. On mentionnera ici le participe "passif» chez Meigret (1980 [1550] : 98) et le «Participe preterit » chez Estienne (1557: 72), pour ne citer qu'eux.

6. Par exemple, « actif » chez Meigret (1980 [1550] : 98), « Participe de temps pres[ent] terminé en ant » chez Maupas (1618, f. 155v). On trouvera un inventaire complet chez Díaz Villalba (2017 : 273-277, 686-687).

7. "Notre langue possède une partie du discours qui ne peut être réduite à aucune des neuf autres. Elle n'existe pas en grec, ni en latin, ni en hébreu, ni non plus en arabe. Comme elle n'a 
pas encore de nom chez nous, nous osons l'appeler nom participial infini: nom, parce qu'elle signifie la substance et elle n'a pas de temps ; participial, parce qu'elle est similaire au participe du temps passé ; infini, parce qu'elle n'a ni genre, ni nombre, ni cas, ni personne déterminés. » (Nous traduisons)

8. «Le supin, étant la forme active de l'infinitif qui se joint, dans tous les verbes, aux auxiliaires ter (avoir) et haver (avoir) pour former les temps composés ou temps par circonlocution, n'a pas de pluriel, ni de terminaison féminine, ce qui le différencie des participes passifs du prétérit: bien que ces derniers soient formés sur lui, ils comportent les deux genres et des terminaisons, car la nature du participe consiste en cela. » (Nous traduisons)

9. Sont exclus les auteurs qui catégorisent le PTC comme un simple participe. Nous avons relevé les étiquettes terminologiques pour les PTC et les sous-classes du participe dans 97 grammaires (Díaz Villalba 2017 : 680-691).

10. Cette sous-série est composée d'influences : Irson s'inspire de Maupas, Vallart (1744:234) reconnaît l'influence de Dangeau, notamment par l'emprunt terminologique de participe auxiliaire, et la RAE se fait l'écho de la proposition de Correas, un auteur que l'institution place dans son horizon de rétrospection ( $c f$. RAE 1771/Sarmiento 1984).

11. «Le participe simple, que nous appelons parfois supin [...] dans les périphrases de la voix active, signifie l'action, si bien que dire yo e amado (j'ai aimé), c'est comme dire « c'est de moi que l'action d'aimer est provenue ». (Nous traduisons)

12. Dans ce cas, le terme nominativo n'a pas le sens traditionnel de nominatif, il désigne le nom.

\section{RÉSUMÉS}

Les formes participiales des temps composés font l'objet d'un traitement particulier chez les grammairiens des différentes langues romanes depuis la Renaissance. Les formes participiales posent en effet un problème assez spécifique, dès lors que dans ces vernaculaires elles présentent des propriétés incompatibles avec la classe du participe telle que la définit la tradition latine. Certains proposent de recatégoriser ces formes en leur affectant une désignation ou une nouvelle classe avec des propriétés plus adaptées. La mise en série des options théoriques relevées dans un corpus étendu (XVe-XVIII ${ }^{\mathrm{e}} \mathrm{s}$.) tend à souligner l'importance de cette manière d'appréhender les données qui mettent à l'épreuve le modèle descriptif latin. Par ailleurs, la récurrence et la commensurabilité des solutions théoriques dans diverses traditions montrent l'intérêt de sortir du cadre des histoires nationales.

The participles of compound tenses have been the subject of special treatment among grammarians of Romance languages since the Renaissance. Such forms raise a fairly specific issue since their properties do not appear to be compatible with the class of participles as the Latin tradition defines it. Some suggest recategorizing these words by giving them a new designation or assigning them to a new class with more suitable properties. This study brings together a series of texts (15th-18th centuries) in which theoretical options are proposed, and it highlights the importance of recategorization for dealing with data that challenge the Latin descriptive model. Furthermore, the recurrence and commensurability of theoretical solutions in various traditions show the interest of going beyond the framework of national histories. 
INDEX

Keywords : categorization, Romances languages, grammar, participle, series of texts, compound tense, grammatical tradition, word class, auxiliary verb

Mots-clés : catégorisation, langues romanes, grammaire, participe, série textuelle, temps composé, tradition grammaticale, classe de mot, verbe auxiliaire

\section{AUTEUR}

\section{ALEJANDRO DÍAZ VILLALBA}

Histoire des théories linguistiques (UMR 7597) 